of the tongue may occur. Rarely spasm of laryngeal and pharyngeal muscles cause choking and respiratory distress. There is no mental impairment, although some patients may initially be considered hysterical due to the unusual appearance of some reactions. ${ }^{4}$ Reactions usually occur within a couple of days of starting treatment, but can also present on drug withdrawal. ${ }^{3}$ Once recognised they should be treated with an anticholinergic such as benztropine or procyclidine, which normally leads to prompt resolution.

A clear history and classical presentation may make the diagnosis evident but the following two clinical histories show that this is not always the case. In both these cases the initial lack of a history of drug ingestion delayed the correct diagnosis, the first being treated as a cervical spine injury and the other as meningitis, with inappropriate and potentially harmful drugs being given.

Accident and emergency (A\&E) staff should consider drug induced dystonic reactions in patients with an appropriate clinical presentation and press for any history of drug ingestion. Many patients are often unaware of the medication they are taking, especially elderly people. Patients who abuse drugs recreationally may deny ingestion because of fear that this information will be passed to police; they should be reassured of the confidential nature of their history. In these cases treatment for possible drug induced dystonic reaction may be diagnostic as well as therapeutic.

\section{Case reports}

CASE 1

A schoolboy of 14 years presented by emergency ambulance to the A\&E department. He had fallen after slipping on ice in the playground and was complaining of neck pain. The ambulancemen had fitted a cervical collar and placed him on a spinal board. On arrival he was treated as a potential cervical spine injury and standard $x$ rays taken. These were normal, and immobilisation measures were discontinued. A torticollis was evident and over the next few minutes he developed a marked extensor spasm of his cervical spine and a fixed upward gaze. He denied taking any drugs but in view of his presentation he was given intravenous benztropine and there was a full resolution of his symptoms and signs. Despite further questioning he maintained that he had not taken any drugs, only a drink of lemonade given to him by a friend at school.

\section{CASE 2}

On the same day a general practitioner in the city was called to see another 14 year old boy at his grandmother's house. He had come home from school early, complaining of a sore neck. There was no history of trauma and he had been perfectly well on leaving home earlier. He was apyrexial and apart from "holding his head to the left side" examination was unremarkable. In view of the boy's distress he was sent to the on call paediatric team for their opinion. Initial assessment noted that he complained of sore neck, feeling numb around the mouth, difficulty in swallowing saliva, and episodes where he was unable to see. While being examined in the admissions unit he developed extensor spasm of his upper body and a fixed upward gaze. He was conscious and noted to have a "grinning" appearance. He was thought to be generally hyperreflexic and possibly to have bilateral papilloedema. Blood was taken for full blood count, C-reactive protein, blood cultures, urea and electrolytes, calcium, magnesiurn, and blood gases. Urine was sent for toxicology and culture. He was given intravenous dexamethasone, mannitol ( $300 \mathrm{ml}$ of $20 \%$ ), and cefotaxime. As spasms continued, after one hour he was given intravenous diazepam, then 15 minutes later procyclidine. Shortly after this the spasms settled but he continued to receive intravenous dexamethasone and cefotaxime until the next day, after which he was observed for a further 24 hours before being discharged. He denied any drug ingestion but it transpired that he had put some of his grandmother's metoclopramide in a bottle of lemonade from which he and his friend (case 1) had drunk. Diagnoses initially considered on presentation had included meningitis and tetanus.

1 Farrell PE, Diehl AK Acute dystonic reaction to crack cocaine. Ann Emerg Med 1991;20:322

2 Brady W, Hall K. Erythromycin related dystonic reaction. Am $\mathcal{F}$ Emerg Med 1992;10:616.

3 Barnes TRE, Edwards JG. In: Antipsychotic drugs and their side-effects. 1993:213-47.

4 Haddad LM, Winchester JF. In: Clinical management of poisoning and drug overdose. 1990:783-4.
South Birmingham Trauma Unit, General hospital, Steelhouse Lane, Birmingham M Taylor A Thoma

Midland Centre for Neurosurgery, Smrthwick, Birmingham A Jackowski Correspondence to: Dr M Taylor, Flat 24, Holly Mount, 291 Hagley Road, Edgbaston, Birmingham

\title{
Missed cervical spine injury following barflying
}

\author{
M Taylor, A Thomas, A Jackowski
}

\author{
Abstract \\ The case is reported of a young woman \\ who suffered a wedge fracture of $\mathrm{C} 7$ due \\ to axial loading with a flexed spine, in an
}

injury caused by barfly jumping. The fracture was unstable and required surgical stabilisation. In this case the seriousness of the injury was not realised at first 
because neck radiographs were not taken at her initial assessment in accident and emergency.

(f Accid Emerg Med 1996;13:222-223)

Key terms: cervical spine fracture; instability; axial loading on flexed spine; barfly injury

\section{Case report}

An 18 year old student was attending the university ball and, after drinking a large quantity of alcohol, had a go at barfly jumping. ${ }^{1}$ The game involves wearing a velcro body suit, jumping off a springboard, somersaulting through the air, and trying to get stuck on a velcro hooked lined wall upside down. Any fall is broken by an inflated crash mattress.

Unfortunately she hit the side of the wall and fell to the ground with a direct blow to her occiput with flexion of her neck. Although she had some neck pain she continued at the party and then went to bed. The following day she attended a casualty department because of persistent neck pain. A diagnosis of a soft tissue injury was made without a cervical spine $x$ ray, and a soft collar was prescribed. She attended her general practitioner the following day at the university health centre, asking for some physiotherapy for her neck. Before a physiotherapy referral, the practitioner arranged a cervical spine $x$ ray, which showed a wedge fracture of $\mathrm{C} 7$ (fig 1). She was then referred to the South Birmingham Trauma Unit and then to the Midland Centre for Neurosurgery.

On examination, she had no neurological signs but any neck movement was limited by pain and muscle spasm.

INVESTIGATIONS

Further investigations by magnetic resonance imaging (MRI) scan and lateral cervical $x$ rays

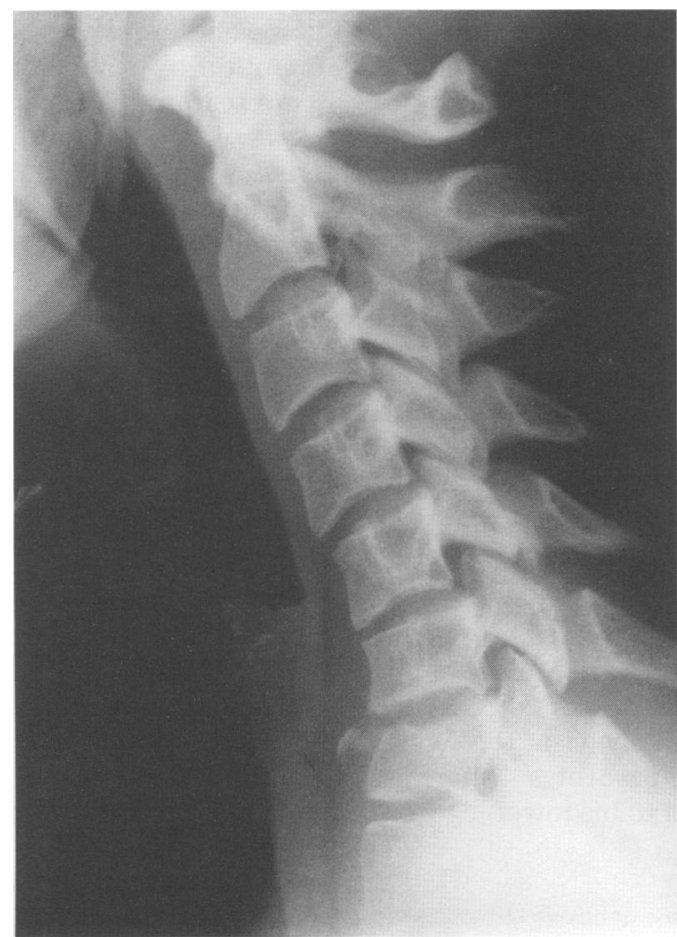

Figure 1 Lateral cervical spine $x$ ray showing wedge fracture of $C 7$

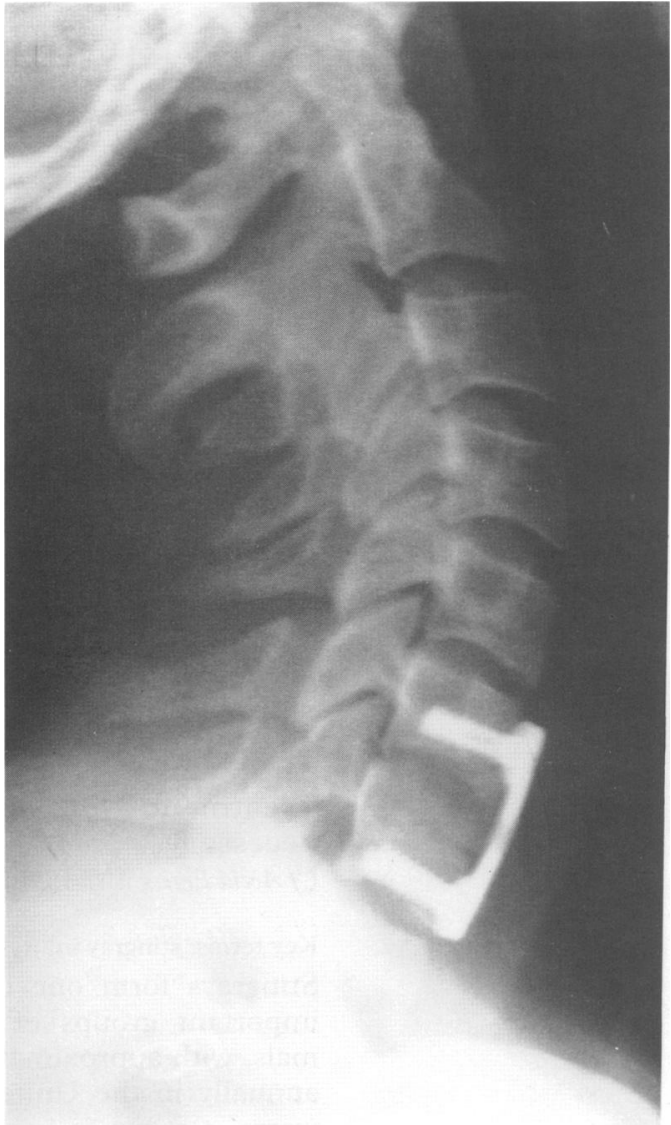

Figure 2 Repair of injury by surgical stabilisation

in flexion and extension were performed. These showed that half the $\mathrm{C} 6 / \mathrm{C} 7$ disc was disrupted, with a wedge fracture of the upper aspect of C7. She had presumably also ruptured her posterior interspinal and supraspinal ligaments, as flexion/extension views revealed gross instability.

\section{TREATMENT}

She underwent an anterior discectomy, removal of loose bony fragments, anterior bone grafting, and insertion of an Ao locking plate across $\mathrm{C} 6 / \mathrm{C} 7$ (fig 2). Recovery from surgery was uneventful and she was allowed home in a semirigid collar one week later.

\section{Discussion}

Although the method of injury is unusual, the mechanism of axial loading with a flexed spine is a common cause of cervical spine injury. The patient's symptoms of persistent midline neck pain and tenderness alerted her general practitioner to the possible seriousness of the injury. The instability of the fracture is demonstrated in the flexion view, with disruption of both the body of $\mathrm{C} 7$ and the posterior interspinous ligaments as shown by the gap between the spinous processes of $\mathrm{C} 6$ and C7. To allow preoperative planning, an MRI scan was performed. This excluded retropulsed bony or disc fragments. As the fracture was unstable, it required surgical stabilisation, as shown in fig 2 .

1 Chesser T, et al. Barfly injury [letter]. BMF 1992;305:583. 